\title{
Dietary fat intakes in Irish adults in 2011: how much has changed in 10 years?
}

\author{
Kaifeng $\mathrm{Li}^{1}$, Breige A. McNulty ${ }^{1}$, Ann M. Tiernery ${ }^{1}$, Niamh F. C. Devlin ${ }^{1}$, Triona Joyce ${ }^{1}$, Joao C. Leite ${ }^{1}$, \\ Albert Flynn ${ }^{2}$, Janette Walton ${ }^{2}$, Lorraine Brennan ${ }^{1}$, Michael J. Gibney ${ }^{1}$ and Anne P. Nugent ${ }^{1 *}$ \\ ${ }^{1}$ School of Agriculture and Food Science, Institute of Food and Health, University College Dublin, Belfield, Dublin 4, Ireland \\ ${ }^{2}$ School of Food and Nutritional Sciences, University College Cork, Cork, Ireland
}

(Submitted 9 June 2015 - Final revision received 1 February 2016 - Accepted 11 February 2016 - First published online 21 March 2016)

\section{Abstract}

Imbalances in dietary fat intakes are linked to several chronic diseases. This study describes dietary intakes and food sources of fat and fatty acids in 1051 Irish adults (aged 18-90 years), using data from the 2011 national food consumption survey, the National Adult Nutrition Survey. It also compares current intakes for 18-64-year-olds with those reported in the last such survey in 2001, the North/South Ireland Food Consumption Survey. Dietary fat intakes were estimated using data from 4-d semi-weighed (2011) and 7-d estimated (2001) food diaries. In 2011, intakes for 18-64-year-olds were as follows: total fat, 34·1 (SD 6.1) \% total energy (\%TE); SFA, 13.3 (sD 3.3) \%TE; MUFA, 12.5 (SD 2.6) \% TE; PUFA, 6.1 (SD 2.2)\%TE; and trans-fat, 0.511 (sD 0.282)\%TE. Apart from MUFA, intakes decreased $(P<0.001)$ compared with 2001. There was no statistically significant difference in intakes of EPA and DHA by 18-64-year-olds in 2011 (269.0 (SD 515.0) mg/d) and 2001 (279.1 (sD 497.5) mg/d). In 2011, adults aged >65 years had the highest intakes of SFA; however, intakes were typically higher than UK-recommended values for all groups. In contrast, intakes of long-chain $n$-3 fatty acids were lowest in younger age groups. Intakes of trans-fat were well within UK-recommended levels. Although there have been some improvements in the profile of intakes since 2001 , imbalances persist in the quantity and quality of dietary fat consumed by Irish adults, most notably for total and SFA and for younger age groups for long-chain $n$-3 fatty acids.

Key words: Dietary fats: Fatty acid intakes: $\boldsymbol{n}$-3 Fatty acids: SFA: Trans-fat

The macronutrient fat is an important source of energy in the diet in addition to being an essential component of cell membranes and precursor to signalling molecules ${ }^{(1)}$. In 1950s, Keys et al. reported that a high-fat diet may lead to CHD and atherosclerosis $^{(2,3)}$. Subsequent research has identified that imbalances in dietary fat intake are related to risk factors for a number of chronic diseases - for example, elevated intakes of SFA increase circulating LDL-cholesterol concentrations and risk of $\mathrm{CVD}^{(4)}$, whereas long-chain $n$-3 PUFA confer benefits to metabolic health ${ }^{(5)}$ and age-related functional decline ${ }^{(6)}$.

On the basis of such evidence, global, regional and national competent organisations or authorities have formulated dietary reference values (DRV) or recommendations to guide public health policy relating to total fat and each of fat type. Globally, the World Health Organization ${ }^{(7)}$ established recommendations for total fat intake of $15-35 \%$ total energy (\%TE), SFA of $<10 \%$ TE, trans-fat of $<1 \% \mathrm{TE}$ and PUFA of $6-10 \% \mathrm{TE}$. Broadly similar guidelines were set by the European Food Safety Authority (EFSA); however, they suggested that intakes of SFA and transfat should be as low as possible, and they have set a recommendation for EPA + DHA at $250 \mathrm{mg} / \mathrm{d}^{(8)}$. In addition, individual countries such as the $\mathrm{UK}^{(9)}$, France ${ }^{(10)}, \mathrm{USA}^{(11)}$, China ${ }^{(12)}$ and Japan $^{(13)}$ have all set national guidelines.

Globally, national food consumption surveys facilitate the analysis of dietary fat intakes and allow monitoring of compliance with the aforementioned dietary guidelines using food intake data collected by a variety of methodologies ${ }^{(14-19)}$. Data from such surveys have typically indicated imbalances in the quality of dietary fat consumed (e.g. excessive intakes of total and/or SFA as reported recently for the UK and Finland) ${ }^{(20,21)}$. This has resulted in various public health education campaigns in Ireland and, for some dietary fats, industry initiatives' and government-industry partnerships to help consumers improve intake profiles such as reducing $\mathrm{SFA}^{(22,23)}$. The last national food consumption survey in Ireland (the North/South Ireland Food Consumption Survey (NSIFCS) ${ }^{(24)}$ ) estimated intakes of total fat and SFA at 36 and $14 \% \mathrm{TE}$, respectively, generally above those recommended ${ }^{(25)}$. The National Adult Nutrition Survey (NANS) ${ }^{(26)}$ was published 10 years later in 2011. The objective of the present study is to characterise dietary fat and fatty acid intakes in Irish adults in 2011 using comprehensive composition data. It will also compare current dietary fat intakes with those

Abbreviations: \%TE; percentage of total energy; ALA; $\alpha$-linolenic acid; EFSA; European Food Safety Authority; NANS; National Adult Nutrition Survey.

* Corresponding author: A. P. Nugent, email anne.nugent@ucd.ie 
reported in 2001 to characterise any changes that may have occurred in fat intakes in the intervening 10 -year period.

\section{Methods \\ Study populations}

The analysis for this paper is based on data from two cross-sectional national food surveys of Irish adults: NANS and NSIFCS ${ }^{(24,26)}$. The NANS (2011) and the NSIFCS (2001) examined the habitual food and beverage consumption of representative samples of Irish adults during the periods 2008-2010 and 1997-1999, respectively (2011: $n$ 1500, 740 men and 760 women aged 18-90 years; 2001: $n$ 1379, 662 men and 717 women aged 18-64 years). The response rates were 60 and $63 \%$ in 2011 and 2001, respectively, with the high level of researcher-participant interaction helping maintain response rates and compliance in both surveys (three to four visits per participant over the survey periods). The sample sizes (1500 and 1379) were chosen to represent a population of over four million people and to deliver at least 100 individuals in the least populated age and sex subgroups. There were few exclusion criteria, other than pregnancy/lactation and inability to complete the survey because of disability. Analysis of both samples has shown them to be representative of the Irish adult population with respect to age, sex, social class and urban/rural location when compared with the relevant Irish censuses, as described by Irish Universities Nutrition Alliance in 2001 and $2011^{(24,26)}$. Both studies were conducted according to the guidelines laid down in the Declaration of Helsinki, with informed consent obtained from all subjects. All procedures involving human subjects were approved by relevant committees, with full details of the sampling procedures and study protocols described elsewhere ${ }^{(27,28)}$.

\section{Food intake assessment}

In 2011, food, beverage and supplement intake was determined using a 4-d semi-weighed food record recorded at brand level where possible. Participants were also asked to retain the packaging of all foods, beverages and supplements consumed. After quantification ${ }^{(26)}$, initial energy and nutrient analysis was conducted using the Weighed Intake Software Program version 3.0 (Tinuviel Software), which uses data from McCance and Widdowson's The Composition of Foods, fifth and sixth editions plus all nine supplemental volumes to generate nutrient intake data, as described elsewhere ${ }^{(26)}$. If necessary, adjustments were made to account for recipes, nutritional supplements, commonly consumed generic Irish foods and new foods to the market. The final food consumption database was initially examined to identify those foods/drinks containing fat. Accompanying databases were created for recipes and for food supplements at brand level. In contrast, the 2001 survey assessed food and nutrient intake using a 7-d estimated food record $^{(24)}$. Although the recording methods differed slightly, similar protocols were followed regarding quantification and assessment of nutrient intakes ${ }^{(24)}$, with a description of intakes of dietary fatty acids over the 7-d period described elsewhere ${ }^{(25,29)}$. The current paper aimed to compare intakes of dietary fat between 2001 and 2011. However, as the dietary records of 2001 were based on a 7 -d estimated food record, the 2001 data were re-allocated from 7-d food record to 4-d food record, and the bootstrap method was used to enable a more direct comparison with the data from $2011^{(21,30)}$. This was carried out by re-sampling 100 independent randomisations using the bootstrap method, and parameter estimates were averaged over 100 bootstrap samples.

\section{Estimation of dietary fat composition of foods}

Methodologies used to calculate the dietary fat composition of foods and recipes consumed in 2001 have previously been described $^{(25,29)}$. A similar protocol was followed for foods and recipes consumed in 2011. First, an SPSS database was created containing all of the foods consumed, including recipes. Each food item in this database was linked to the original consumption database using a unique food code. Each food code was then examined and assigned a fatty acid concentration for total fat, SFA, MUFA, PUFA, $\alpha$-linolenic acid (ALA), EPA, DHA and trans-fat $(\mathrm{g} / 100 \mathrm{~g})$ based on published food composition tables ${ }^{(31-33)}$, published papers ${ }^{(25,29)}$, the packaging information collected during NANS and with updated industry data included where available. Published values were used for EPA and DHA contents of milk and milk products and some meats, as values are typically absent from food composition tables despite being present in low concentrations in these commonly consumed foods ${ }^{(29)}$. A similar approach was adopted for the recipe database whereby the fatty acid composition was obtained for each ingredient as described above and the values were aggregated before re-introduction into main databases.

\section{Calculating fatty acid intakes of Irish adults}

The total fat, SFA, MUFA, PUFA, EPA, DHA and trans-fat contents ( $\mathrm{g} / 100 \mathrm{~g}$ product) were available for $n 2552$ foods and recipes in 2011 and 2938 in 2001. ALA intakes were estimated only in 2011; they were not available for 2001. All entries were double-checked by the researcher, and a random sample was checked by another researcher in-house to minimise the chance of error. To represent the actual amount of fatty acid consumed, each fatty acid concentration in the database was converted from $\mathrm{g} / 100 \mathrm{~g}$ food to grams per actual weight of food consumed. Information from this database was then used to calculate the mean daily intake of each fatty acid per person per day.

\section{Data analysis}

Under-reporters of energy intake (20-30\% of total populations) were identified as having a ratio of energy intake:BMR of $<1.05^{(34)}$ and were excluded in this analysis; hence, the final sample sizes were 1051 (2011) and 1097 (2001) individuals. Data were analysed using SPSS version 20 (SPSS Inc.). The mean values and standard deviations of total fat, SFA, MUFA, PUFA, ALA, EPA, DHA and trans-fat intakes (g/d and as \%TE) were calculated for the total population in 2011 by sex and age group (18-35, 36-50, 51-64 and over 65 years). To calculate 
compliance levels for trans-fat, intakes were expressed as percentage contribution to food energy (\%FE; energy content from alcohol was excluded as per relevant recommendation). When examined, the data for individual fatty acid intakes are skewed, but because of the large sample size parametric tests are allowed to be applied (based on the central limit theorem). Differences in fat intake accounting for age, survey and sex were detected either using independent $t$ tests, one-way ANOVA (age group) or a two-way ANOVA (age and survey) as appropriate. $P$ values are presented uncorrected for multiple testing, because of the high expected correlation of the tests. We encourage readers to examine the effect sizes presented, and furthermore we note which results would survive a conservative Bonferroni correction. Foods consumed in 2011 and 2001 were aggregated into twenty-two different food categories, and the percentage contribution from each food group to total fatty acid intake was calculated as population proportions $^{(35)}$. As there are no national dietary guidelines for Ireland, compliance with the dietary guidelines from the $\mathrm{UK}^{(9,36)}$ and $\mathrm{EFSA}^{(8)}$ (for EPA + DHA) was assessed by estimating the maximal size of subgroup of 'compliers' who met the population target ${ }^{(37)}$. Briefly, this method involves ranking individual mean daily intakes from lowest to highest. For the $\%$ TE from total fat and SFA recommendations, the mean intake of the compliers group was calculated by starting with the individual with the lowest mean \%TE from fat (or SFA) and including successive individuals until the addition of one more subject led to the average intake of the group to exceed the DRV $^{(37)}$. The same approach was used for MUFA, PUFA and $\mathrm{EPA}+\mathrm{DHA}$, except that mean daily intakes for each individual were ranked in descending order from highest to lowest and successive individuals added until the addition of the next individual caused the group mean to fall below the population target. The percentage of adults who were 'compliers' with each recommendation was then calculated.

\section{Results}

The final NANS sample comprised $49 \cdot 8 \%$ men and $50 \cdot 2 \%$ women and included $65.2,19.4$ and $15.4 \%$ in the age groups $18-50,51-64$ and $\geq 65$ years, respectively. The majority of the sample comprised individuals from an urban location (68.9\%), and almost half ( $46 \cdot 1 \%)$ of them were classified as professionals or in technical or managerial occupations. The final sample was representative of the Irish population in terms of age, sex, education level and location ${ }^{(38)}$. The demographic characteristics of the NSIFCS have been described in detail elsewhere and were also representative of the Irish population at that time ${ }^{(39)}$. The mean energy intakes were 9347 (sD 2623) kJ (2234 (sD 627) kcal) and 10192 (sD 2987) kJ (2436 (sD 714) kcal) in 2010 and 2001, respectively.

\section{Mean daily intake of dietary fat in 2011 and 2001 by age and sex}

Tables 1-4 present the mean daily intakes of dietary fat in $\mathrm{g} / \mathrm{d}$ and as \%TE in Irish adults, by age and sex in 2011 with comparison with 2001 as relevant. In 2011, intake of total fat (\%TE) was 34.1 (SD 6.1) \% for adults aged 18-64 years and 34.9 (sD 6.9$) \%$ for those aged more than 65 years $(P=0.136)$, with intakes higher $(P<0.001)$ in women aged $18-64$ years $(34.9$ (SD 5.9)\%) than in men (33.3 (SD 6.2)\%). Compared with 2001 (35.7 (SD 5.9)\%TE), lower intakes of total fat were observed in 2011 for 18-64-year-olds and for men and women of all age categories $(P<0 \cdot 001)$ (Tables 3 and 4). In 2011, dietary intakes (\%TE) of SFA in the total population were 13.3 (SD 3.3 ) \% for 18-64-year-olds, with higher $(P=0 \cdot 003)$ intakes for those aged more than 65 years (14.3 (SD 4.1)\%). A similar pattern emerged whereby higher intakes of SFA (\%TE) were recorded in 2001 than in 2011 for the total population aged 18-64 years (13.9 (sD 3.4) $v .13 \cdot 3$ (sD 3.3) \%, $P<0.001)$ and for men and women of all ages, except men aged 51-64 years where a slight increase was observed. However, after Bonferroni correction (for fourteen to sixteen tests), only significant differences in SFA intakes (\%TE) between 2011 and 2001 for the total population and for women aged 18-64 years remained significant at a family-wise type I error rate of $5 \%$. In 2011, intakes (\%TE) of MUFA were 12.5 (SD 2.6) and 12.2 (SD 2.9) \% for adults aged 18-64 years and over 65 years, respectively. Higher $(P=0.017)$ intakes were observed for women (12.7 (SD 2.6)\%) aged 18-64 years compared with men $(12 \cdot 3$ (SD $2 \cdot 7) \%$ ), but intakes were higher $(P \leq 0.002)$ in all population groups in 2011 than in 2001. In 2011, intakes of PUFA (\%TE) were $6 \cdot 1$ (SD $2 \cdot 2$ ) \% for 18-64 years and 5.9 (SD 2.5$) \%$ for over 65 years in the total population. Intakes of PUFA were higher in women than in men $(P \leq 0 \cdot 027)$, but with lower intakes in 2011 than 2001 for all population groups $(P<0.001)$. After Bonferroni correction (for sixteen tests), only differences in PUFA intakes between the 2 time points remained significant at a family-wise type I error rate of $5 \%$.

In 2011, ALA intakes (\%TE) were 0.60 (sD 0.39) \% for those aged 18-64 years and 0.59 (SD 0.76) \% for those aged more than 65 years in the total population, with intakes significantly higher in women than in men in both age categories $(P<0 \cdot 027)$. In 2011, the mean daily intakes of EPA and DHA from food and supplements (mg/d) were 113 (sD 244.3) and 156 (sD 284.3) mg/d for adults aged 18-64 years, respectively, with significantly higher $(P<0.001)$ intakes observed in adults aged over 65 years (187.9 (sD 261.1) and 247.7 (sD 300.5) mg/d, respectively). There was no difference in intakes between the surveys; however, in both instances the lowest intakes were observed for men and women aged 18-35 years. In 2011, combined intakes of EPA and DHA for men and women aged 18-35 years were 160.3 (SD 240.4) and 201.8 (sD 299.3) mg/d. Significantly $(P<0.001)$ higher intakes were reported for supplement users than non-users in 2011, but not in $2001(P>0 \cdot 001$, see online Supplementary Table S1). The contribution (\%) of trans-fat intake to total energy intake was 0.51 (SD 0.28) \% for adults aged 18-64 years and 0.56 (SD 0.36 )\% for adults aged more than 65 years. Intakes of trans-fat (\%TE) were slightly higher in men than in women aged 18-64 years $(0.53$ (SD 0.30$) v .0 .49$ (SD 0.26) \%, $P=0.039)$ and in 2001 than in $2011(0.69$ (SD 0.25) $v$. 0.51 (SD 0.28)\%, $P<0.001$ ). After Bonferroni correction (for sixteen tests), only differences in trans-fat intakes between the 2 time points remained significant at a family-wise type I error rate of $5 \%$. 
Table 1. Intakes of total fat ( $\mathrm{g} / \mathrm{d}$ and percentage of total energy (\%TE)) and its constituent fatty acids in Irish adults in 2011 (Mean values and standard deviations)

\begin{tabular}{|c|c|c|c|c|c|c|c|c|c|c|c|c|c|c|c|}
\hline & \multicolumn{15}{|c|}{2011} \\
\hline & \multicolumn{4}{|c|}{ Total population } & \multirow[b]{3}{*}{$P^{*}$} & \multirow{2}{*}{\multicolumn{2}{|c|}{$\begin{array}{c}\text { Male } \\
\begin{array}{c}18-64 \text { years } \\
(n \text { 448) }\end{array}\end{array}$}} & \multirow{2}{*}{\multicolumn{2}{|c|}{$\begin{array}{c}\text { Female } \\
\begin{array}{c}\text { 18-64 years } \\
(n \text { 441) }\end{array}\end{array}$}} & \multirow[b]{3}{*}{$P \dagger$} & \multicolumn{2}{|c|}{ Male } & \multicolumn{2}{|c|}{ Female } & \multirow[b]{3}{*}{$P \ddagger$} \\
\hline & \multicolumn{2}{|c|}{$\begin{array}{c}18-64 \text { years } \\
(n 889)\end{array}$} & \multicolumn{2}{|c|}{$\begin{array}{c}\geq 65 \text { years } \\
(n 162)\end{array}$} & & & & & & & \multicolumn{2}{|c|}{$\geq 65$ years $(n 75)$} & \multicolumn{2}{|c|}{$\geq 65$ years $(n 87)$} & \\
\hline & Mean & SD & Mean & SD & & Mean & SD & Mean & SD & & Mean & SD & Mean & SD & \\
\hline Total fat $(\mathrm{g} / \mathrm{d})$ & $86 \cdot 8$ & 27.9 & $74 \cdot 3$ & $29 \cdot 3$ & $<0.001$ & 98.6 & 28.9 & 74.9 & $20 \cdot 8$ & $<0.001$ & 84.5 & $35 \cdot 1$ & 65.5 & 19.5 & $<0.001$ \\
\hline SFA $(g / d)$ & 33.9 & $12 \cdot 4$ & 30.7 & 14.9 & 0.004 & 38.7 & 13.0 & 28.9 & $9 \cdot 4$ & $<0.001$ & $35 \cdot 6$ & $17 \cdot 9$ & 26.5 & 10.0 & $<0.001$ \\
\hline MUFA (g/d) & 31.9 & $11 \cdot 1$ & 26.0 & 10.6 & $<0.001$ & $36 \cdot 4$ & 11.5 & $27 \cdot 4$ & 8.5 & $<0.001$ & 29.8 & $12 \cdot 3$ & $22 \cdot 6$ & 7.5 & $<0.001$ \\
\hline PUFA (g/d) & $15 \cdot 4$ & $6 \cdot 8$ & $12 \cdot 4$ & 5.9 & $<0.001$ & $16 \cdot 9$ & 7.5 & 13.9 & 5.5 & $<0.001$ & $13 \cdot 1$ & $6 \cdot 2$ & $11 \cdot 7$ & 5.6 & 0.137 \\
\hline $\operatorname{ALA}(\mathrm{g} / \mathrm{d})$ & 1.5 & 0.9 & $1 \cdot 2$ & 1.6 & 0.002 & 1.7 & 1.0 & 1.4 & 0.9 & $<0.001$ & 1.1 & 0.6 & 1.3 & $2 \cdot 1$ & 0.350 \\
\hline $\operatorname{EPA}(\mathrm{mg} / \mathrm{d})$ & 113.4 & 244.3 & 187.9 & $261 \cdot 1$ & $<0.001$ & $125 \cdot 0$ & $306 \cdot 1$ & $101 \cdot 7$ & $158 \cdot 1$ & 0.154 & 184.2 & $222 \cdot 6$ & 191.2 & 291.5 & 0.865 \\
\hline $\mathrm{DHA}(\mathrm{mg} / \mathrm{d})$ & 155.6 & 284.3 & 247.7 & 300.5 & $<0.001$ & 162.9 & 329.3 & 148.2 & 229.8 & 0.442 & 265.8 & 314.4 & 232.2 & 289.0 & 0.480 \\
\hline Trans-fat (g/d) & 1.3 & 0.9 & 1.2 & 0.9 & 0.135 & 1.6 & 1.0 & 1.1 & 0.6 & $<0.001$ & 1.4 & 1.1 & 1.0 & 0.7 & 0.009 \\
\hline Total fat (\%TE) & 34.1 & $6 \cdot 1$ & 34.9 & 6.9 & 0.136 & 33.3 & $6 \cdot 2$ & 34.9 & 5.9 & $<0.001$ & 34.7 & $7 \cdot 3$ & 35.1 & 6.6 & 0.693 \\
\hline SFA (\%TE) & 13.3 & 3.3 & 14.3 & 4.1 & 0.003 & 13.1 & 3.2 & 13.5 & 3.3 & 0.052 & 14.5 & 4.4 & 14.2 & 4.0 & 0.606 \\
\hline MUFA (\%TE) & 12.5 & 2.6 & $12 \cdot 2$ & $2 \cdot 9$ & 0.153 & $12 \cdot 3$ & $2 \cdot 7$ & $12 \cdot 7$ & 2.6 & 0.017 & $12 \cdot 3$ & 3.0 & 12.1 & $2 \cdot 8$ & 0.719 \\
\hline PUFA (\%TE) & $6 \cdot 1$ & $2 \cdot 2$ & 5.9 & 2.5 & 0.375 & 5.7 & $2 \cdot 1$ & 6.5 & $2 \cdot 3$ & $<0.001$ & 5.4 & 1.8 & $6 \cdot 3$ & 3.0 & 0.027 \\
\hline ALA (\%TE) & 0.60 & 0.39 & 0.59 & 0.76 & 0.724 & 0.56 & 0.38 & 0.64 & 0.39 & 0.002 & 0.46 & 0.19 & 0.70 & 1.01 & 0.027 \\
\hline EPA (\%TE) & 0.046 & 0.091 & 0.093 & 0.126 & $<0.001$ & 0.044 & 0.103 & 0.048 & 0.076 & 0.445 & 0.080 & 0.105 & 0.103 & 0.141 & 0.255 \\
\hline DHA (\%TE) & 0.064 & 0.114 & 0.124 & 0.151 & $<0.001$ & 0.057 & 0.115 & 0.070 & 0.112 & 0.075 & 0.114 & 0.138 & 0.131 & 0.161 & 0.478 \\
\hline Trans-fat (\%TE) & 0.511 & 0.282 & 0.558 & 0.360 & 0.116 & 0.530 & 0.304 & 0.491 & 0.256 & 0.039 & 0.567 & 0.373 & 0.550 & 0.351 & 0.777 \\
\hline
\end{tabular}

$n$, Number of participants; ALA, $a$-linolenic acid.

* Independent samples $t$ test for comparison of means between age groups.

† Independent samples $t$ test for comparison of means between sex.

‡ Independent samples $t$ test for comparison of means between National Adult Nutrition Survey and North/South Ireland Food Consumption Survey. 
Table 2. Intakes of total fat ( $\mathrm{g} / \mathrm{d}$ and percentage of total energy (\%TE)) and its constituent fatty acids in Irish adults in 2001 (Mean values and standard deviations)

\begin{tabular}{|c|c|c|c|c|c|c|c|c|c|}
\hline & \multicolumn{9}{|c|}{2001} \\
\hline & \multicolumn{2}{|c|}{ Total population } & \multirow[b]{3}{*}{$P^{*}$} & \multicolumn{2}{|c|}{ Male } & \multirow[b]{3}{*}{$P \dagger$} & \multicolumn{2}{|c|}{ Female } & \multirow[b]{3}{*}{$P \ddagger$} \\
\hline & \multicolumn{2}{|c|}{$18-64$ years $(n 1097)$} & & \multicolumn{2}{|c|}{$18-64$ years $(n 555)$} & & \multicolumn{2}{|c|}{$18-64$ years $(n 542)$} & \\
\hline & Mean & SD & & Mean & SD & & Mean & SD & \\
\hline Total fat $(\mathrm{g} / \mathrm{d})$ & $96 \cdot 7$ & $32 \cdot 8$ & $<0.001$ & $111 \cdot 2$ & $34 \cdot 1$ & $<0.001$ & $81 \cdot 8$ & $23 \cdot 6$ & $<0.001$ \\
\hline SFA $(g / d)$ & 37.9 & $15 \cdot 4$ & $<0.001$ & 43.7 & $16 \cdot 6$ & $<0.001$ & $32 \cdot 0$ & $11 \cdot 3$ & $<0.001$ \\
\hline MUFA (g/d) & $32 \cdot 4$ & 11.5 & 0.372 & 37.5 & 11.8 & 0.142 & $27 \cdot 2$ & 8.4 & 0.669 \\
\hline PUFA (g/d) & $18 \cdot 9$ & 7.9 & $<0.001$ & 21.5 & 8.6 & $<0.001$ & $16 \cdot 4$ & $6 \cdot 2$ & $<0.001$ \\
\hline $\operatorname{ALA}(g / d)$ & - & - & - & - & - & - & - & - & - \\
\hline EPA (mg/d) & $124 \cdot 3$ & 234.7 & 0.314 & $125 \cdot 5$ & 223.1 & 0.977 & $123 \cdot 1$ & $246 \cdot 2$ & 0.099 \\
\hline $\mathrm{DHA}(\mathrm{mg} / \mathrm{d})$ & $154 \cdot 8$ & 265.4 & 0.952 & $160 \cdot 5$ & $257 \cdot 1$ & 0.898 & $149 \cdot 1$ & $273 \cdot 7$ & 0.958 \\
\hline Trans-fat (g/d) & 1.9 & 0.9 & $<0.001$ & $2 \cdot 1$ & 1.0 & $<0.001$ & 1.6 & 0.7 & $<0.001$ \\
\hline Total fat (\%TE) & $35 \cdot 7$ & $5 \cdot 9$ & $<0.001$ & $35 \cdot 0$ & $6 \cdot 0$ & $<0.001$ & $36 \cdot 4$ & $5 \cdot 7$ & $<0.001$ \\
\hline SFA (\%TE) & $13 \cdot 9$ & $3 \cdot 4$ & $<0.001$ & $13 \cdot 7$ & 3.4 & 0.005 & $14 \cdot 2$ & $3 \cdot 4$ & 0.002 \\
\hline MUFA (\%TE) & 11.9 & $2 \cdot 2$ & $<0.001$ & $11 \cdot 8$ & $2 \cdot 3$ & 0.002 & $12 \cdot 1$ & $2 \cdot 2$ & $<0.001$ \\
\hline PUFA (\%TE) & $7 \cdot 1$ & $2 \cdot 3$ & $<0.001$ & 6.8 & $2 \cdot 2$ & $<0.001$ & $7 \cdot 3$ & $2 \cdot 3$ & $<0.001$ \\
\hline ALA (\%TE) & - & - & - & - & - & - & - & - & - \\
\hline EPA (\%TE) & 0.049 & 0.098 & 0.497 & 0.042 & 0.078 & 0.744 & 0.056 & 0.114 & 0.200 \\
\hline DHA (\%TE) & 0.061 & 0.110 & 0.562 & 0.053 & 0.089 & 0.595 & 0.068 & 0.128 & 0.762 \\
\hline Trans-fat (\%TE) & 0.686 & 0.253 & $<0.001$ & 0.673 & 0.255 & $<0.001$ & 0.698 & 0.250 & $<0.001$ \\
\hline
\end{tabular}

$n$, Number of participants; ALA, $a$-linolenic acid; -, data not available.

* Independent samples $t$ test for comparison of means between age groups.

$\dagger$ Independent samples $t$ test for comparison of means between sex.

¥ Independent samples $t$ test for comparison of means between National Adult Nutrition Survey and North/South Ireland Food Consumption Survey.

Table 3. Intakes of total fat ( $\mathrm{g} / \mathrm{d}$ and percentage of total energy (\%TE)) and its constituent fatty acids in Irish men in 2011 and 2001 by age group (Mean values and standard deviations)

\begin{tabular}{|c|c|c|c|c|c|c|c|c|c|c|c|c|c|c|c|}
\hline & \multicolumn{6}{|c|}{2011} & \multicolumn{6}{|c|}{2001} & & & \\
\hline & \multicolumn{2}{|c|}{$\begin{array}{c}\text { 18-35 years } \\
(\text { ( } 207)\end{array}$} & \multicolumn{2}{|c|}{$\begin{array}{c}\text { 36-50 years } \\
(n 143)\end{array}$} & \multicolumn{2}{|c|}{$\begin{array}{c}\text { 51-64 years } \\
(n 98)\end{array}$} & \multicolumn{2}{|c|}{$\begin{array}{c}18-35 \text { years } \\
(n 218)\end{array}$} & \multicolumn{2}{|c|}{$\begin{array}{c}36-50 \text { years } \\
(n 198)\end{array}$} & \multicolumn{2}{|c|}{$\begin{array}{c}\text { 51-64 years } \\
(n 139)\end{array}$} & \multicolumn{3}{|c|}{ Two-way ANOVA } \\
\hline & Mean & SD & Mean & SD & Mean & SD & Mean & SD & Mean & SD & Mean & SD & A & S & $A \times S$ \\
\hline Total fat $(\mathrm{g} / \mathrm{d})$ & 102.4 & $27 \cdot 9$ & $96 \cdot 8$ & $30 \cdot 1$ & 93.4 & $28 \cdot 7$ & $116 \cdot 8$ & 34.5 & $113 \cdot 3$ & $34 \cdot 2$ & 99.4 & 30.5 & $<0.001$ & $<0.001$ & 0.128 \\
\hline SFA (g/d) & 39.5 & $13 \cdot 2$ & $38 \cdot 6$ & $12 \cdot 4$ & 37.4 & 13.5 & $45 \cdot 6$ & 16.5 & $45 \cdot 0$ & $17 \cdot 3$ & 38.9 & $14 \cdot 6$ & 0.001 & $<0.001$ & 0.099 \\
\hline MUFA $(g / d)$ & 38.3 & 11.0 & $35 \cdot 6$ & $12 \cdot 6$ & 33.6 & $10 \cdot 2$ & 39.7 & $12 \cdot 3$ & 37.7 & 11.5 & 33.7 & 10.5 & $<0.001$ & 0.109 & 0.582 \\
\hline PUFA (g/d) & 17.9 & $6 \cdot 6$ & $16 \cdot 2$ & 7.6 & $15 \cdot 9$ & 8.9 & $23 \cdot 1$ & 8.7 & 21.6 & 8.3 & $18 \cdot 8$ & 8.3 & $<0.001$ & $<0.001$ & 0.139 \\
\hline $\operatorname{ALA}(g / d)$ & 1.8 & 0.7 & 1.6 & 1.4 & 1.5 & 0.9 & - & - & - & - & - & - & 0.041 & - & - \\
\hline EPA (mg/d) & $86 \cdot 4$ & $135 \cdot 7$ & 113.5 & $196 \cdot 8$ & 223.4 & $568 \cdot 1$ & $92 \cdot 9$ & 148.4 & $137 \cdot 0$ & $240 \cdot 2$ & $160 \cdot 3$ & 282.4 & $<0.001$ & 0.519 & 0.133 \\
\hline $\mathrm{DHA}(\mathrm{mg} / \mathrm{d})$ & $115 \cdot 4$ & $177 \cdot 1$ & 149.7 & 268.4 & 282.2 & $555 \cdot 3$ & 124.4 & 184.4 & 174.2 & 276.0 & 197.5 & 315.4 & $<0.001$ & 0.367 & 0.064 \\
\hline Trans-fat (g/d) & 1.7 & 1.1 & 1.5 & 0.8 & 1.5 & 1.0 & 2.1 & 1.0 & 2.3 & 1.1 & 2.0 & 0.9 & 0.057 & $<0.001$ & 0.082 \\
\hline Total fat (\%TE) & $32 \cdot 7$ & $6 \cdot 2$ & 33.8 & 5.8 & 33.8 & 6.8 & $35 \cdot 0$ & $5 \cdot 7$ & 35.5 & $6 \cdot 0$ & $34 \cdot 1$ & $6 \cdot 2$ & 0.158 & $<0.001$ & 0.118 \\
\hline SFA (\%TE) & $12 \cdot 6$ & $3 \cdot 2$ & 13.5 & 3.0 & 13.5 & 3.4 & $13 \cdot 6$ & 3.2 & 14.0 & 3.5 & $13 \cdot 3$ & 3.6 & 0.026 & 0.047 & 0.067 \\
\hline MUFA (\%TE) & $12 \cdot 3$ & $2 \cdot 7$ & $12 \cdot 4$ & $2 \cdot 6$ & $12 \cdot 2$ & $2 \cdot 8$ & 11.9 & $2 \cdot 2$ & 11.8 & $2 \cdot 2$ & 11.6 & $2 \cdot 4$ & 0.575 & 0.001 & 0.723 \\
\hline PUFA (\%TE) & $5 \cdot 7$ & 1.9 & $5 \cdot 7$ & $2 \cdot 1$ & $5 \cdot 7$ & $2 \cdot 7$ & $7 \cdot 0$ & $2 \cdot 2$ & 6.9 & $2 \cdot 3$ & $6 \cdot 4$ & $2 \cdot 2$ & 0.296 & $<0.001$ & 0.224 \\
\hline ALA (\%TE) & 0.57 & 0.20 & 0.58 & 0.59 & 0.52 & 0.25 & - & - & - & - & - & - & 0.41 & - & - \\
\hline EPA (\%TE) & 0.028 & 0.043 & 0.041 & 0.076 & 0.081 & 0.186 & 0.028 & 0.046 & 0.045 & 0.082 & 0.058 & 0.104 & $<0.001$ & 0.296 & 0.155 \\
\hline DHA (\%TE) & 0.037 & 0.058 & 0.053 & 0.103 & 0.104 & 0.188 & 0.039 & 0.058 & 0.057 & 0.093 & 0.071 & 0.117 & $<0.001$ & 0.169 & 0.068 \\
\hline Trans-fat (\%TE) & 0.533 & 0.321 & 0.529 & 0.276 & 0.526 & 0.310 & 0.639 & 0.228 & 0.709 & 0.268 & 0.676 & 0.271 & 0.267 & $<0.001$ & 0.186 \\
\hline
\end{tabular}

$n$, Number of participants; A, age; S, survey; ALA, $a$-linolenic acid; -, data not available.

The percentage of Irish adults adhering to different dietary fat recommendations

The percentage of Irish adults adhering to dietary recommendations of the UK Department of Health ${ }^{(9)}$ for total fat, SFA and MUFA, EFSA recommendations for ALA, EPA and DHA ${ }^{(8)}$, and UK Scientific Advisory Committee on Nutrition (SACN) ${ }^{(36)}$ recommendations for trans-fat in 2011 is presented in Table 5.
High levels of compliance were noted for adults aged 18-64 years and those aged over 65 years for total fat (94, 86\%), MUFA (100, $100 \%)$, PUFA (100, 98\%), ALA (100, 100\%) and trans-fat (100, $100 \%)$. By examining intakes from supplements and foods, high levels of compliance were observed for adults aged 18-64 and >65 years for EPA and DHA (100, 100\%). Lower rates of compliance were observed when intakes from food only were assessed, with the lowest levels of compliance 
Table 4. Intakes of total fat ( $\mathrm{g} / \mathrm{d}$ and percentage of total energy (\%TE)) and its constituent fatty acids in Irish women in 2011 and 2001 by age group (Mean values and standard deviations)

\begin{tabular}{|c|c|c|c|c|c|c|c|c|c|c|c|c|c|c|c|}
\hline & \multicolumn{6}{|c|}{2011} & \multicolumn{6}{|c|}{2001} & & & \\
\hline & \multicolumn{2}{|c|}{$\begin{array}{c}18-35 \text { years } \\
(n 170)\end{array}$} & \multicolumn{2}{|c|}{$\begin{array}{c}36-50 \text { years } \\
(n 165)\end{array}$} & \multicolumn{2}{|c|}{$\begin{array}{c}51-64 \text { years } \\
(n 106)\end{array}$} & \multicolumn{2}{|c|}{$\begin{array}{c}18-35 \text { years } \\
(n \text { 206) }\end{array}$} & \multicolumn{2}{|c|}{$\begin{array}{c}36-50 \text { years } \\
(n \text { 224) }\end{array}$} & \multicolumn{2}{|c|}{$\begin{array}{c}\text { 51-64 years } \\
(n 112)\end{array}$} & \multicolumn{3}{|c|}{ Two-way ANOVA } \\
\hline & Mean & SD & Mean & SD & Mean & SD & Mean & SD & Mean & SD & Mean & SD & A & S & $A \times S$ \\
\hline Total fat $(\mathrm{g} / \mathrm{d})$ & 78.7 & 22.9 & $72 \cdot 8$ & 19.5 & 71.9 & $18 \cdot 6$ & $82 \cdot 7$ & $23 \cdot 2$ & $82 \cdot 7$ & $24 \cdot 1$ & 78.4 & $23 \cdot 2$ & 0.011 & $<0.001$ & 0.190 \\
\hline SFA $(g / d)$ & 29.9 & 9.8 & $28 \cdot 6$ & $9 \cdot 3$ & $27 \cdot 9$ & 8.9 & $32 \cdot 3$ & $11 \cdot 3$ & 31.9 & $11 \cdot 3$ & $31 \cdot 3$ & 11.5 & 0.228 & $<0.001$ & 0.814 \\
\hline MUFA $(g / d)$ & $29 \cdot 4$ & $9 \cdot 2$ & $26 \cdot 4$ & 7.8 & $25 \cdot 8$ & $7 \cdot 6$ & 27.5 & 8.0 & $27 \cdot 6$ & 8.9 & $25 \cdot 8$ & $7 \cdot 8$ & 0.001 & 0.669 & 0.040 \\
\hline PUFA (g/d) & 14.8 & 5.8 & $13 \cdot 2$ & 5.0 & 13.5 & 5.7 & $16 \cdot 9$ & $6 \cdot 3$ & $16 \cdot 6$ & 6.0 & 14.9 & $6 \cdot 1$ & 0.004 & $<0.001$ & 0.091 \\
\hline $\operatorname{ALA}(\mathrm{g} / \mathrm{d})$ & 1.4 & 0.7 & 1.3 & 1.0 & 1.4 & 0.8 & - & - & - & - & - & - & 0.768 & - & - \\
\hline EPA (mg/d) & 62.9 & $100 \cdot 7$ & $104 \cdot 7$ & $157 \cdot 4$ & 159.1 & 209.5 & 79.9 & 181.9 & $119 \cdot 0$ & 231.5 & $210 \cdot 6$ & 339.8 & $<0.001$ & 0.045 & 0.529 \\
\hline $\mathrm{DHA}(\mathrm{mg} / \mathrm{d})$ & 97.5 & $148 \cdot 4$ & 156.9 & $236 \cdot 4$ & 216.0 & 299.4 & $102 \cdot 1$ & 202.5 & 144.1 & $262 \cdot 3$ & $245 \cdot 3$ & 371.1 & $<0.001$ & 0.671 & 0.613 \\
\hline Trans-fat (g/d) & $1 \cdot 1$ & 0.6 & 1.0 & 0.5 & 1.0 & 0.6 & 1.6 & 0.7 & 1.6 & 0.7 & 1.6 & 0.8 & 0.772 & $<0.001$ & 0.783 \\
\hline Total fat (\%TE) & $35 \cdot 0$ & $5 \cdot 7$ & 34.9 & $6 \cdot 1$ & 34.6 & $6 \cdot 1$ & 36.6 & $5 \cdot 3$ & $36 \cdot 6$ & 5.9 & 35.6 & $6 \cdot 1$ & 0.305 & $<0.001$ & 0.739 \\
\hline SFA (\%TE) & $13 \cdot 3$ & $3 \cdot 1$ & $13 \cdot 7$ & $3 \cdot 3$ & $13 \cdot 5$ & 3.6 & $14 \cdot 3$ & 3.5 & $14 \cdot 1$ & $3 \cdot 3$ & $14 \cdot 1$ & 3.4 & 0.943 & 0.003 & 0.512 \\
\hline MUFA (\%'TE) & $13 \cdot 0$ & 2.5 & $12 \cdot 6$ & 2.5 & $12 \cdot 4$ & 2.7 & $12 \cdot 1$ & 1.9 & $12 \cdot 2$ & 2.4 & 11.7 & $2 \cdot 3$ & 0.029 & $<0.001$ & 0.461 \\
\hline PUFA (\%TE) & 6.5 & 1.9 & $6 \cdot 4$ & 2.5 & 6.5 & $2 \cdot 4$ & 7.5 & $2 \cdot 3$ & 7.4 & $2 \cdot 3$ & $6 \cdot 8$ & $2 \cdot 3$ & 0.120 & $<0.001$ & 0.116 \\
\hline ALA (\%TE) & 0.63 & 0.29 & 0.65 & 0.49 & 0.65 & 0.36 & - & - & - & - & - & - & 0.858 & - & - \\
\hline EPA (\%TE) & 0.027 & 0.044 & 0.051 & 0.080 & 0.076 & 0.099 & 0.036 & 0.082 & 0.054 & $0 \cdot 107$ & 0.096 & 0.160 & $<0.001$ & 0.103 & 0.563 \\
\hline DHA (\%TE) & 0.043 & 0.065 & 0.077 & 0.120 & 0.103 & 0.144 & 0.046 & 0.091 & 0.066 & 0.124 & 0.113 & 0.175 & $<0.001$ & 0.960 & 0.521 \\
\hline Trans-fat (\%TE) & 0.485 & 0.245 & 0.488 & 0.245 & 0.506 & 0.290 & 0.693 & 0.252 & 0.696 & 0.242 & 0.712 & 0.264 & 0.618 & $<0.001$ & 0.999 \\
\hline
\end{tabular}

$n$, Number of participants; A, age; S, survey; ALA, $a$-linolenic acid; -, data not available.

for men (58\%) and women (47\%) aged $18-35$ years. Relatively low levels of compliance persisted for SFA (36 and 30\%TE in 18-64-year-olds and >65-year-olds, respectively), with the lowest levels of compliance observed for men and women aged $36-50$ years (27 and $30 \%$ ) and men aged $>65$ years (27\%). By comparison, the proportions of adults aged 18-64 years meeting the recommendations were higher in 2011 than in 2001 for all dietary fats and for all population groups, except for EPA and DHA (see online Supplementary Table S2).

\section{Percentage contribution of food groups to dietary fat in 2011 and 2001}

The percentage contribution of food groups to dietary fat in 2011 and 2001 are presented in Tables 6 and 7. 'Fresh meat' made the greatest contribution to total fat, SFA and MUFA in both 2011 and 2001, followed by 'meat products', 'biscuits, cakes, pastries and buns' and 'spreading fats and oils'. Whole milk featured more strongly as a contributor to SFA intakes in 2001 than in 2011. In 2011, the main contributors to PUFA were 'meat products' and 'potatoes chipped, fried and roasted', whereas in 2001 the major contributors were 'spreading fats and oils' and 'potatoes chipped, fried and roasted'. 'Meat dishes' and 'spreading fats and oils' were the greatest contributors to ALA intake in 2011 (Table 7). The major food sources of EPA and DHA were 'fish, fish products and fish dishes' and 'fresh meat' in both 2011 and 2001. Unsurprisingly, 'nutritional supplements' featured as a contributor to EPA and DHA when intakes from food supplements were included in the estimation of intakes. The main contributors to trans-fat were 'cheeses', 'fresh meat', 'meat dishes' and 'whole milk' in 2011, whereas in 2001 they were 'cheeses', 'fresh meat' and 'butter'.

\section{Discussion}

Over the decade 2001-2011, improvements in the profile of dietary fats consumed by Irish adults have been observed. However, imbalances in dietary fat quality remain whereby intakes of SFA were higher for all population groups than recommended and with the lowest intakes of long-chain $n$-3 PUFA for young men and women. Population intakes of trans-fat were within recommended levels. This analysis shows that in 2011 population intakes of total fat (both $\mathrm{g} / \mathrm{d}$ and \%TE), SFA (both $\mathrm{g} / \mathrm{d}$ and \%TE), PUFA (both $\mathrm{g} / \mathrm{d}$ and \%TE) and trans-fat (both $\mathrm{g} / \mathrm{d}$ and \%TE) decreased compared with the previous national food survey in 2001; intakes of MUFA (\%TE) increased, whereas no change was evident for EPA and DHA.

Intakes of SFA (13\%TE) by Irish adults in 2011 agree with those reported in other EU countries such as France, Finland or the UK $(12-15 \% \mathrm{TE})^{(8,40)}$, albeit higher compared with other global regions such as North and South America, Asia and Africa where intakes can lie $\leq 10 \% \mathrm{TE}^{(14,15,41)}$. Although a slight reduction of $0.6 \%(P<0.001)$ in mean daily intakes has occurred in Ireland since 2001, average intakes of SFA in Ireland continue to deviate from such numerical guidelines of about $10 \% \mathrm{TE}^{(9)}$ and from the EFSA guideline of 'as low as possible ${ }^{,(8)}$. This poses challenges, as despite public health education campaigns and initiatives by the food industry and regulators all population groups were affected, with the lowest levels of compliance $(<29 \%)$ with the $<10 \%$ TE recommendation $^{(9)}$ by older men aged $>65$ years and men and women aged 36-50 years. Currently, the major food sources of SFA continue to be fresh meats, whole milk and cheeses, as well as meat products and 'biscuits, cakes, pastries and buns', which together contribute $40 \%$ to SFA intakes, similar to that reported for other European countries ${ }^{(21,42)}$. Collectively, reductions in the 
contributions of whole milk and butter to SFA intakes in adults aged 18-64 years (by approximately $-6 \%$ from 2001) may reflect public health messages ${ }^{(23,43)}$ to choose lower-fat options; however, butter remains a greater contributor for older (8.4\%) than younger $(5.3 \%)$ adults. Hence, intakes of SFA continue to deviate from current guidance in Ireland. However, this combination of foods contributing to SFA intakes is noteworthy (e.g. milks, meats, biscuits and cakes). Consideration of these differing foods may now be merited in any future dietary guidelines given the growing body of evidence, which suggests differential effects of SFA on health depending on the food form in which they occur ${ }^{(44)}$.

In this study, intakes of trans-fat have decreased by approximately $0 \cdot 2 \%$ energy in the decade from 2001 to 2011 and, at $0.5 \% \mathrm{TE}(0.5 \% \mathrm{FE})$, are well below the UK $\mathrm{SACN}^{(36)}$ recommendation of $\leq 2 \% \mathrm{FE}$, similar to that reported for Finland $(0.4 \% \mathrm{TE})^{(20)}$, albeit slightly lower than that reported for the UK $(0.8 \% \mathrm{FE})^{(21)}$. The major dietary sources of trans-fat in the Irish adult diet are now ruminant animal products such as meat, cheese and whole milk ( $42 \%$ contribution), with decreases observed for foods such as 'spreading fats and oils' (from 5.9 to $1.4 \%$ intakes). This is significant given that intakes of trans-fats from animal sources have not been related to CHD events ${ }^{(41)}$ in contrast to those from industrial sources ${ }^{(42)}$. It also suggests that commercial foods containing artificial trans-fats are no longer on sale, or at least are no longer consumed to a significant degree, in Ireland.

In general, mean daily intakes of EPA and DHA $(269 \mathrm{mg} / \mathrm{d})$ in Irish adults from food and supplements met the EFSArecommended adequate intake (AI) of $\geq 250 \mathrm{mg} / \mathrm{d}^{(8)}$. Mean daily intakes from food and supplements reported here were in line with mean values published for a sample of Belgian women $(276 \mathrm{mg} / \mathrm{d})^{(45)}$, with median values (range $71-228 \mathrm{mg} / \mathrm{d}$ ) broadly comparable with published median intakes for a representative sample of Belgian adults ${ }^{(46)}$. However, median values were higher than those observed for the Dutch National Food Survey depending on age and $\operatorname{sex}^{(47)}$. Intakes from food alone were marginally higher in Irish adults; this is perhaps because of the methodology used here, which factored for $n-3$ content of milks and some meats ${ }^{(29)}$. Nevertheless, a distribution of intakes was evident in Irish adults, whereby the highest mean daily intakes from all sources were recorded for adults aged $>65$ years $(436(\mathrm{sD} 542) \mathrm{mg} / \mathrm{d})$ and the lowest intakes for men (202 (sD 299) $\mathrm{mg} / \mathrm{d}$ ) and women (161 (sD 240) $\mathrm{mg} / \mathrm{d}$ ) aged 18-35 years. Given the beneficial role of these fatty acids for cardiovascular health and particularly during pregnancy and lactation (where EFSA recommend increasing intakes of DHA by an additional $\left.100-200 \mathrm{mg} / \mathrm{d}^{(8)}\right)$, such low intakes by younger subgroups may have implications for longer-term health. Among consumers, supplements contributed up to $55 \%$ of total EPA+ DHA intake. Furthermore, the use of $n-3$ supplements was higher in $2011(12.5 \%)$ than in 2001 (7.5\%) for all (see online Supplementary Table S2). Upon exclusion of supplement users, levels of compliance with the recommended AI of $\geq 250 \mathrm{mg} / \mathrm{d}^{(8)}$ dropped to $85 \%$ of the total population, with lowest compliance (47\%) for female non-supplement users aged 18-35 years. Unsurprisingly, 'fish, fish products and fish dishes' and 'fresh meat' were the main food contributors in the population, which 
Table 6. Percentage contribution of food groups to total fat, SFA, MUFA and PUFA in Irish adults in 2011 and 2001 (excluding contribution from supplements)

\begin{tabular}{|c|c|c|c|c|c|c|c|c|c|c|c|c|}
\hline & \multicolumn{3}{|c|}{ Total fat } & \multicolumn{3}{|c|}{ SFA } & \multicolumn{3}{|c|}{ MUFA } & \multicolumn{3}{|c|}{ PUFA } \\
\hline & \multicolumn{2}{|c|}{2011} & \multirow{2}{*}{$\frac{2001}{\frac{18-64 \text { years }}{(n \text { 1097) }}}$} & \multicolumn{2}{|c|}{2011} & \multirow{2}{*}{$\frac{2001}{2 \begin{array}{c}18-64 \text { years } \\
(n 1097)\end{array}}$} & \multicolumn{2}{|c|}{2011} & \multirow{2}{*}{$\frac{2001}{\frac{18-64 \text { years }}{(n 1097)}}$} & \multicolumn{2}{|c|}{2011} & \multirow{2}{*}{$\begin{array}{c}2001 \\
\begin{array}{c}18-64 \text { years } \\
(n 1097)\end{array}\end{array}$} \\
\hline & $\begin{array}{l}\text { 18-64 years } \\
(n 889)\end{array}$ & $\begin{array}{l}\geq 65 \text { years } \\
(n 162)\end{array}$ & & $\begin{array}{l}\text { 18-64 years } \\
(n \text { 889) }\end{array}$ & $\begin{array}{l}\geq 65 \text { years } \\
(n 162)\end{array}$ & & $\begin{array}{l}\text { 18-64 years } \\
(n 889)\end{array}$ & $\begin{array}{l}\geq 65 \text { years } \\
(n 162)\end{array}$ & & $\begin{array}{l}\text { 18-64 years } \\
(n \text { 889) }\end{array}$ & $\begin{array}{l}\geq 65 \text { years } \\
(n 162)\end{array}$ & \\
\hline Fresh meat & $9 \cdot 1$ & $13 \cdot 0$ & 9.9 & $8 \cdot 7$ & $12 \cdot 3$ & $9 \cdot 7$ & $10 \cdot 7$ & $15 \cdot 6$ & $12 \cdot 6$ & 7.5 & $11 \cdot 2$ & $6 \cdot 8$ \\
\hline Meat products & 8.4 & $6 \cdot 1$ & $7 \cdot 2$ & 7.5 & 5.6 & $7 \cdot 0$ & $10 \cdot 2$ & 7.6 & 8.8 & $8 \cdot 2$ & $6 \cdot 2$ & 5.4 \\
\hline Meat dishes & 4.7 & $4 \cdot 3$ & 4.9 & 4.9 & 4.7 & $4 \cdot 3$ & $5 \cdot 0$ & 3.7 & $5 \cdot 4$ & 3.6 & $3 \cdot 7$ & $4 \cdot 3$ \\
\hline Fish, fish products and fish dishes & $3 \cdot 0$ & $5 \cdot 1$ & $3 \cdot 1$ & 1.6 & $2 \cdot 7$ & 1.7 & $3 \cdot 3$ & 5.9 & 3.6 & 4.7 & $8 \cdot 3$ & 4.7 \\
\hline Egg and egg dishes & $2 \cdot 9$ & $3 \cdot 1$ & $2 \cdot 6$ & 2.5 & $2 \cdot 4$ & $2 \cdot 1$ & 3.3 & 3.8 & $3 \cdot 1$ & $2 \cdot 2$ & $2 \cdot 6$ & 1.9 \\
\hline Spreading fats and oils & 8.4 & $12 \cdot 1$ & 11.5 & $8 \cdot 1$ & $10 \cdot 0$ & 8.8 & 8.9 & $12 \cdot 8$ & 9.7 & $10 \cdot 1$ & $17 \cdot 1$ & $20 \cdot 8$ \\
\hline Butter & 3.4 & $6 \cdot 2$ & $5 \cdot 2$ & $5 \cdot 3$ & 8.4 & 7.8 & $2 \cdot 4$ & 4.8 & $4 \cdot 0$ & 0.8 & $2 \cdot 1$ & $1 \cdot 3$ \\
\hline Potatoes chipped, fried and roasted & 5.4 & $2 \cdot 6$ & 5.9 & $3 \cdot 3$ & 1.5 & 3.5 & $6 \cdot 3$ & 3.2 & $6 \cdot 8$ & $8 \cdot 1$ & 3.8 & $10 \cdot 1$ \\
\hline Potato products & 1.0 & 0.5 & 0.8 & 0.5 & 0.2 & 0.5 & 0.7 & 0.4 & 0.6 & $2 \cdot 4$ & 1.6 & 1.9 \\
\hline Potatoes & 0.8 & 1.2 & $1 \cdot 2$ & $1 \cdot 1$ & 1.5 & $1 \cdot 2$ & 0.5 & 0.8 & 0.7 & 0.9 & 1.3 & $2 \cdot 2$ \\
\hline Breads and rolls & $5 \cdot 3$ & 5.8 & 4.9 & 4.8 & 4.7 & 3.7 & 3.5 & 4.1 & 3.8 & 5.8 & 7.5 & $7 \cdot 0$ \\
\hline Biscuits, cakes, pastries and buns & $7 \cdot 1$ & 7.4 & $7 \cdot 1$ & $8 \cdot 3$ & $8 \cdot 3$ & $8 \cdot 2$ & $7 \cdot 3$ & $7 \cdot 8$ & 7.5 & 5.5 & $6 \cdot 1$ & $4 \cdot 3$ \\
\hline $\begin{array}{l}\text { Sugars, confectionery and } \\
\text { preserves }\end{array}$ & $4 \cdot 1$ & 1.6 & $3 \cdot 6$ & $5 \cdot 9$ & $2 \cdot 3$ & 4.9 & $4 \cdot 1$ & 1.6 & $3 \cdot 8$ & 1.5 & $0 \cdot 7$ & $1 \cdot 1$ \\
\hline Puddings and chilled desserts & $2 \cdot 1$ & 3.3 & 3.0 & 3.1 & 4.8 & 3.8 & 1.8 & 2.9 & $2 \cdot 9$ & 1.0 & 1.5 & 1.4 \\
\hline Ice cream and cream & $1 \cdot 3$ & 1.8 & 1.7 & $2 \cdot 2$ & $2 \cdot 8$ & $2 \cdot 7$ & 0.9 & $1 \cdot 2$ & 1.4 & 0.3 & 0.3 & 0.4 \\
\hline Cheeses & $5 \cdot 1$ & 4.2 & 4.3 & 8.0 & $6 \cdot 2$ & $6 \cdot 8$ & 4.0 & 3.5 & 3.7 & 1.5 & 1.3 & 1.0 \\
\hline Whole milk & 4.6 & $4 \cdot 3$ & 6.5 & $7 \cdot 0$ & $6 \cdot 3$ & $10 \cdot 2$ & $3 \cdot 3$ & $3 \cdot 3$ & 5.8 & 0.8 & 0.8 & 1.0 \\
\hline Low-fat, skimmed and fortified milk & 1.7 & $2 \cdot 7$ & 1.6 & $2 \cdot 7$ & $4 \cdot 2$ & $2 \cdot 7$ & 1.5 & 2.5 & 1.5 & 0.0 & 0.0 & 0.0 \\
\hline Savouries (e.g. pizza, quiche) & 3.8 & 1.2 & $2 \cdot 7$ & 3.9 & $1 \cdot 1$ & $2 \cdot 4$ & 3.9 & $1 \cdot 3$ & $3 \cdot 1$ & 3.5 & $1 \cdot 3$ & $3 \cdot 3$ \\
\hline Savoury snacks (e.g. crisps) & $2 \cdot 6$ & 0.3 & $2 \cdot 2$ & 1.0 & 0.1 & $2 \cdot 2$ & $3 \cdot 1$ & 0.3 & $2 \cdot 6$ & 5.9 & 0.7 & $2 \cdot 0$ \\
\hline Vegetable and pulse dishes & $2 \cdot 8$ & 1.9 & 2.5 & 1.5 & $1 \cdot 2$ & 1.2 & 2.5 & 1.9 & $2 \cdot 0$ & $5 \cdot 9$ & $3 \cdot 1$ & $5 \cdot 8$ \\
\hline Nuts and seeds, herbs and spices & 1.9 & $1 \cdot 1$ & 0.7 & 0.9 & 0.4 & 0.3 & $2 \cdot 2$ & 1.0 & 0.9 & 3.4 & $2 \cdot 4$ & $1 \cdot 2$ \\
\hline $\begin{array}{l}\text { Soups, sauces and miscellaneous } \\
\text { foods }\end{array}$ & 4.9 & $3 \cdot 2$ & $3 \cdot 6$ & $2 \cdot 6$ & $2 \cdot 2$ & $2 \cdot 2$ & $6 \cdot 0$ & $3 \cdot 7$ & $3 \cdot 1$ & $7 \cdot 6$ & $5 \cdot 4$ & $6 \cdot 4$ \\
\hline RTEBC & 1.0 & 0.8 & 0.7 & 0.6 & 0.5 & 0.4 & 1.0 & 0.8 & 0.5 & 1.7 & 1.5 & $1 \cdot 2$ \\
\hline Others* & 4.7 & $6 \cdot 2$ & 2.5 & 4.0 & 5.4 & 1.6 & 3.5 & 4.7 & 1.9 & 7.3 & $9 \cdot 1$ & 4.4 \\
\hline
\end{tabular}

$n$, Number of participants; RTEBC, ready-to-eat breakfast cereals.

* Other food groups include rice, pasta, flours, grains, other breakfast cereals, non-alcoholic beverages, nuts and seeds, herbs and spices, fruit, vegetables, other milk and milk-based beverages. 
Table 7. Percentage contribution of food groups to $a$-linolenic acid (ALA), EPA, DHA and trans-fat intakes in Irish adults in 2011 and 2001 (excluding contribution from supplements)

\begin{tabular}{|c|c|c|c|c|c|c|c|c|c|c|c|c|}
\hline & \multicolumn{3}{|c|}{ ALA } & \multicolumn{3}{|c|}{ EPA } & \multicolumn{3}{|c|}{ DHA } & \multicolumn{3}{|c|}{ Trans-fat } \\
\hline & \multicolumn{2}{|c|}{2011} & \multirow{2}{*}{$\frac{2001}{\begin{array}{c}18-64 \text { years } \\
(n \text { 1097) }\end{array}}$} & \multicolumn{2}{|c|}{2011} & \multirow{2}{*}{$\frac{2001}{2 \begin{array}{c}18-64 \text { years } \\
(n 1097)\end{array}}$} & \multicolumn{2}{|c|}{2011} & \multirow{2}{*}{$\frac{2001}{\begin{array}{c}18-64 \text { years } \\
(n 1097)\end{array}}$} & \multicolumn{2}{|c|}{2011} & \multirow{2}{*}{$\frac{2001}{2} \begin{array}{c}18-64 \text { years } \\
(n 1097)\end{array}$} \\
\hline & $\begin{array}{l}\text { 18-64 years } \\
(n \text { 889) }\end{array}$ & $\begin{array}{c}\geq 65 \text { years } \\
(n 162)\end{array}$ & & $\begin{array}{l}\text { 18-64 years } \\
\quad(n \text { 889) }\end{array}$ & $\begin{array}{c}\geq 65 \text { years } \\
(n 162)\end{array}$ & & $\begin{array}{l}\text { 18-64 years } \\
(n \text { 889) }\end{array}$ & $\begin{array}{c}\geq 65 \text { years } \\
(n 162)\end{array}$ & & $\begin{array}{l}\text { 18-64 years } \\
\quad(n \text { 889) }\end{array}$ & $\begin{array}{c}\geq 65 \text { years } \\
(n 162)\end{array}$ & \\
\hline Fresh meat & $6 \cdot 8$ & $7 \cdot 1$ & - & $25 \cdot 2$ & 23.4 & $21 \cdot 3$ & $20 \cdot 1$ & $17 \cdot 2$ & $26 \cdot 1$ & 14.4 & 18.4 & $10 \cdot 2$ \\
\hline Meat products & 1.4 & $1 \cdot 3$ & - & $2 \cdot 7$ & $2 \cdot 3$ & 3.8 & 0.0 & 0.0 & $6 \cdot 2$ & 5.1 & 4.0 & $7 \cdot 3$ \\
\hline Meat dishes & 11.0 & $10 \cdot 0$ & - & 0.1 & 0.0 & $7 \cdot 2$ & 0.1 & 0.2 & $6 \cdot 0$ & 11.3 & 7.9 & $6 \cdot 1$ \\
\hline Fish, fish products and fish dishes & 3.4 & $5 \cdot 1$ & - & 33.6 & $47 \cdot 7$ & 31.3 & 38.5 & $52 \cdot 4$ & 35.9 & 0.3 & 0.5 & 1.1 \\
\hline Egg and egg dishes & 1.9 & 1.8 & - & 1.4 & $1 \cdot 6$ & 0.3 & $15 \cdot 9$ & $9 \cdot 7$ & $9 \cdot 3$ & 0.8 & 1.4 & 2.6 \\
\hline Spreading fats and oils & 11.5 & $12 \cdot 8$ & - & 3.6 & 3.3 & $12 \cdot 2$ & 0.0 & 0.0 & 0.3 & 1.4 & 1.6 & 5.9 \\
\hline Butter & 8.0 & $6 \cdot 9$ & - & 9.2 & $8 \cdot 1$ & 5.5 & $2 \cdot 4$ & 2.0 & $2 \cdot 8$ & 5.6 & $6 \cdot 1$ & $10 \cdot 7$ \\
\hline Potatoes chipped, fried and roasted & $2 \cdot 1$ & $2 \cdot 1$ & - & 0.0 & 0.0 & 0.0 & 0.0 & 0.0 & 0.0 & 0.6 & 0.6 & $2 \cdot 3$ \\
\hline Potato products & $8 \cdot 1$ & $5 \cdot 8$ & - & 1.9 & 1.5 & 0.1 & $6 \cdot 4$ & 5.9 & 0.1 & 0.5 & 0.6 & 0.7 \\
\hline Potatoes & 0.0 & 0.0 & - & 4.0 & $2 \cdot 2$ & 0.2 & 0.0 & 0.0 & 0.0 & $1 \cdot 1$ & 0.9 & $2 \cdot 1$ \\
\hline Breads and rolls & 0.8 & 1.1 & - & 0.0 & 0.0 & 0.3 & 0.0 & 0.0 & 0.7 & 1.1 & 1.0 & 4.0 \\
\hline Biscuits, cakes, pastries and buns & $2 \cdot 0$ & 1.9 & - & 0.1 & 0.0 & 0.6 & 0.6 & 0.3 & 1.6 & $5 \cdot 8$ & $5 \cdot 1$ & 8.6 \\
\hline $\begin{array}{l}\text { Sugars, confectionery and } \\
\text { preserves }\end{array}$ & $1 \cdot 5$ & $1 \cdot 2$ & - & 0.0 & 0.0 & 0.0 & 0.0 & 0.0 & 0.0 & $5 \cdot 1$ & $5 \cdot 0$ & 1.7 \\
\hline Puddings and chilled desserts & 1.5 & $2 \cdot 1$ & - & 1.6 & 1.1 & 1.3 & 1.5 & 1.7 & 1.2 & 5.7 & $7 \cdot 1$ & 3.6 \\
\hline Ice cream and cream & 0.6 & 0.5 & - & 1.4 & 0.2 & 0.7 & 0.1 & 0.0 & 0.4 & 2.6 & $2 \cdot 7$ & $2 \cdot 3$ \\
\hline Cheeses & 1.0 & 0.8 & - & 8.7 & 5.4 & 4.4 & 11.0 & 8.8 & $2 \cdot 7$ & $16 \cdot 9$ & $17 \cdot 2$ & $10 \cdot 8$ \\
\hline Whole milk & $5 \cdot 2$ & $5 \cdot 2$ & - & 1.9 & $1 \cdot 2$ & $6 \cdot 8$ & 0.3 & 0.1 & 3.8 & $11 \cdot 1$ & $8 \cdot 6$ & $6 \cdot 6$ \\
\hline Low-fat, skimmed and fortified milk & 1.0 & 0.7 & - & 0.0 & 0.0 & 1.8 & 0.1 & 0.0 & 0.6 & 5.4 & $6 \cdot 2$ & 3.8 \\
\hline Savouries (e.g. pizza, quiche) & $2 \cdot 7$ & 3.4 & - & 1.2 & 0.5 & 0.6 & 0.9 & 0.5 & 1.4 & 2.6 & $2 \cdot 3$ & 2.0 \\
\hline Savoury snacks (e.g. crisps) & $2 \cdot 0$ & 1.9 & - & 0.0 & 0.0 & 0.0 & 0.0 & 0.0 & 0.0 & 0.1 & 0.1 & 0.5 \\
\hline Vegetable and pulse dishes & 4.9 & $6 \cdot 1$ & - & 0.7 & $1 \cdot 1$ & 0.5 & 0.3 & 0.6 & 0.6 & 0.6 & 0.9 & 1.3 \\
\hline Nuts and seeds, herbs and spices & 2.6 & 0.7 & - & 0.0 & 0.0 & 0.0 & 0.0 & 0.0 & 0.0 & 0.0 & 0.0 & 0.0 \\
\hline $\begin{array}{l}\text { Soups, sauces and miscellaneous } \\
\text { foods }\end{array}$ & $9 \cdot 0$ & 8.6 & - & 1.4 & 0.3 & 0.4 & 0.7 & 0.5 & 0.3 & 0.8 & 1.1 & $4 \cdot 6$ \\
\hline RTEBC & 1.5 & $2 \cdot 0$ & - & 0.0 & 0.0 & 0.0 & 0.0 & 0.0 & 0.0 & 0.0 & 0.0 & 0.1 \\
\hline Others* & 8.9 & $10 \cdot 4$ & - & 0.6 & 0.1 & 0.3 & 0.4 & 0.1 & 0.3 & 1.0 & 0.6 & 0.7 \\
\hline Nutritional supplements $\dagger$ & 0.7 & 0.6 & - & $5 \cdot 7$ & $10 \cdot 6$ & $4 \cdot 2$ & 4.0 & $6 \cdot 6$ & 3.6 & - & - & - \\
\hline
\end{tabular}

$n$, Number of participants; RTEBC, ready-to-eat breakfast cereals.

* Other food groups include rice, pasta, flours, grains, other breakfast cereals, non-alcoholic beverages, nuts and seeds, herbs and spices, fruit, vegetables, other milk and milk-based beverages.

† Dietary intakes from food and food supplements were included in the analysis of the contribution of food supplements to total population ALA, EPA and DHA intakes. 
is similar to the $\mathrm{UK}^{(21)}$ and other EU countries ${ }^{(42)}$. However, only $52.6 \%$ of Irish consumers (data not shown) eat fish in any form, and in 2011 intake of fish, fish products and dishes was higher in older than in younger adults (data not shown) and in supplement users than in non-users. These patterns were not observed in 2001. This suggests that there is a segment of the Irish population who do not consume fish or fish oil supplements and with inadequate intakes of beneficial long-chain $n$ - 3 fatty acids.

In 2011 , average population intakes of total fat $(34 \cdot 1 \% \mathrm{TE})$ were within EFSA recommendations ${ }^{(8)}$ of $<35 \%$ TE based on the practical considerations and evidence from intervention studies, with intakes higher than reported most recently for Finland or the $\mathrm{UK}^{(20,21)}$. In 2011, the major contributing food groups to total fat intake were dairy products, fresh meat, meat products and biscuits, cakes, pastries and buns, which is similar to 2001 Irish data and to other EU countries ${ }^{(42)}$ and the $\mathrm{USA}^{(11)}$. Average intakes of PUFA by Irish adults in 2011 met the UK's recommendation at $\geq 6 \% \mathrm{TE}^{(9)}$, albeit intakes were slightly lower in men of all ages (approximately 5.7\%). Intakes of PUFA by Irish 18-64-year-olds have declined slightly (1\%) since 2001, as has been reported elsewhere ${ }^{(21,48)}$. Coupled with the observed reduction in SFA intakes, this suggests that reductions in SFA intakes do not automatically result in an increase in PUFA intakes, as observed elsewhere ${ }^{(44)}$. Of note, replacement of SFA with PUFA, rather than MUFA or carbohydrates, is deemed to prevent $\mathrm{CHD}$ and to reduce $\mathrm{CHD}$ incidence over a wide range of intakes ${ }^{(49,50)}$. Hence, any future initiatives to reduce SFA intakes should consider strategies for its replacement.

The strength of this study relates to the quality of the data collected in two national food consumption surveys using estimated and semi-weighed food records, with statistical account given to the differing durations of data recording periods. Particular attention was given to obtaining the most up-to-date fatty acid composition data for foods by availing of updated food composition tables and, as available, analytical data provided by regulatory bodies and the food industry in Ireland. Nevertheless, these data were not available for all foods, and data were not included for the $n$ - 6 PUFA; these limitations must be acknowledged. Future studies should estimate intakes of $n-6$ PUFA to complement the dietary intake data. Other limitations include the self-reported nature of the food diaries, the observational study design and the exclusion of $20-30 \%$ of the original study populations because of their classification as under-reporters. Nevertheless, levels of underreporting were similar to that reported elsewhere ${ }^{(21)}$, and their exclusion of under-reporters deemed important to avoid reporting trends based on increasing levels of under-reporting rather than true changes in fat intake, as reported elsewhere ${ }^{(51)}$. Levels of under-reporting had increased from $20 \%$ in 2001 to $30 \%$ in 2011 , with the profile of under-reporters more likely to be male and slightly older in 2011 than in 2001 (48\% male in 2011 v. 38\% in 2001; mean age 45 (SD 16) v. 42 (sD 12) years). Other limitations relate to intakes of less frequently consumed fatty acid such as EPA and DHA, which may have been underestimated given the short study duration ( $4 \mathrm{~d}$ ), where at least $15 \mathrm{~d}$ of dietary recording for men and $30 \mathrm{~d}$ for women are recommended to account for variations in PUFA intakes ${ }^{(52,53)}$. Hence, a true distribution of usual intakes typically requires intake estimates over many days or the use of statistical modelling. Usual intakes were not assessed in this paper; however, by assessing intakes over 4 survey days, this will partly offset any overestimation in the proportion of people above and below the extremes of intake. Nevertheless, this paper provides a comprehensive estimate of dietary fat and fatty acid intakes in Irish adults using up-to-date composition information, which can be used to inform food-based dietary guidelines and public health policy.

In conclusion, improvements in dietary fat intakes in Irish adults were observed with significant improvements in the direction of dietary recommendations during the last decade; however, the possibility remains for improvement in intakes of SFA and PUFA. Future strategies to modulate population dietary fat intakes should consider not only the quantity and quality of fat consumed across the life span, but also the nature of the contributing foods involved and whether replacement strategies are needed when suggesting reductions in intakes of any one fat type.

\section{Acknowledgements}

The authors thank the Food Safety Authority of Ireland and members of Food and Drink Industry Ireland for assistance with obtaining up-to-date fatty acid composition data. The authors also thank the survey participants for providing detail of their dietary intakes. The authors acknowledge the assistance of Ricardo Segurado (statistician at CStar, www.CStar.ie) for his advice on statistical components of the study.

This research was funded by the Irish Department of Agriculture, Food and the Marine and the Health Research Board under their joint Food for Health Research Initiative (2007-2012) (grant no. FHRIUCC2). K. L. is in receipt of a PhD student from the China Scholarship Council 2012-2016. Funding agencies had no role in the design, analysis or writing of this article.

K. L., B. A. M. and A. P. N. analysed and interpreted the data. K. L. and A. P. N. wrote the manuscript. B. A. M., A. M. T., N. F. C. D., T. J. and J. C. L. prepared the data for analysis. J. W., A. F., M. J. G., B. A. M. and A. P. N. contributed to survey design and data collection and were principal investigators for NANS; A. F. and M. J. G. were principal investigators for NSIFCS. All authors approved the final version of the draft before publication.

There are no conflicts of interest to declare.

\section{Supplementary material}

For supplementary material/s referred to in this article, please visit http://dx.doi.org/doi:10.1017/S0007114516000787

\section{References}

1. Calder PC (2011) Fatty acids and inflammation: the cutting edge between food and pharma. Eur J Pharmacol 668, S50-S58.

2. Keys A, Mickelsen O, Miller EVO, et al. (1950) The relation in man between cholesterol levels in the diet and in the blood. Science 112, 79-81.

3. Keys A (1954) Diet and coronary disease. Lancet 264, 37-38. 
4. Sanders TAB (2013) Reappraisal of SFA and cardiovascular risk Proc Nutr SOC 72, 390-398.

5. Calder PC (2015) Functional roles of fatty acids and their effects on human health. J Parenter Enteral Nutr 39, 18S-32S.

6. Burlingame B, Nishida C, Uauy R, et al. (2009) Fats and fatty acids in human nutrition: introduction. Ann Nutr Metab 55, 5-7.

7. World Health Organization (2003) Diet, Nutrition and the Prevention of Chronic Diseases. Joint WHO/FAO Expert Consultation. WHO Technical Report Series, no. 916. Geneva: WHO.

8. European Food Safety Authority (EFSA) Panel on Dietetic Products, Nutrition, and Allergies (NDA) (2010) Scientific opinion on dietary reference values for fats, including saturated fatty acids, polyunsaturated fatty acids, monounsaturated fatty acids, trans fatty acids, and cholesterol. EFSA J 8, 1461-1568

9. Department of Health (1991) Dietary Reference Values for Food Energy and Nutrients for the United Kingdom. Report of the Panel on Dietary Reference Values of the Committee on Medical Aspects of Food Policy. London: HMSO.

10. Agence Française de Sécurité Sanitaire des Aliments (2001) Apports Nutritionnels Conseillés Pour la Population Française (Recommended Dietary Intake for the French Population). Paris: Editions Tec\&Doc.

11. Institute of Medicine (2005) Dietary Reference Intakes for Energy, Carbohydrate, Fiber, Fat, Fatty Acids, Cholesterol, Protein, and Amino Acids. Washington, DC: National Academies Press.

12. Chinese Nutrition Society (2000) Chinese dietary reference intake, DRIs. Acta Nutrimenta Sinica 23, 193-196.

13. Sasaki S (2008) Dietary reference intakes (DRIs) in Japan. Asia Pac J Clin Nutr 17, 420-444.

14. US Department of Agriculture (2012) What we eat in America, NHANES 2009-2010. http://www.ars.usda.gov/SP2UserFiles/ Place/12355000/pdf/0910/Table_1_NIN_GEN_09.pdf (accessed June 2015).

15. Health Canada, Statistics Canada (2009) Canadian Community Health Survey. Nutrient Intakes from Food: Provincial, Regional and National Data Tables (CCHS Cycle 2.2), Vols 1-3. Ottawa: Health Canada Publications. http://www. hc-sc.gc.ca/fn-an/surveill/nutrition/commun/art-nutr-adult-eng. php (accessed June 2015).

16. McLennan W \& Podger A (1995) National Nutrition Survey Nutrient Intakes and Physical Measurements. Canberra: Australian Bureau Of Statistics. http://www.ausstats.abs.gov.au/ ausstats/subscriber.nsf/0/CA25687100069892CA25688900268A6D/ \$File/48050_1995.pdf (accessed June 2015).

17. University of Otago \& Ministry of Health (2011) A Focus on Nutrition. Key Findings of the 2008/09 New Zealand Adult Nutrition Survey. Wellington: University of Otago \& Ministry of Health. http://www.health.govt.nz/system/files/documents/ publications/a-focus-on-nutrition-v2.pdf (accessed June 2015).

18. Jianzhang LM, Yuanrong J, Chunrong W, et al. (2009) The dietary food intakes and their food sources among Chinese adults. Acta Nutrimenta Sinica 31, 424-427.

19. Ueshima H, Stamler J, Elliott P, et al. (2007) Food omega-3 fatty acid intake of individuals (total, linolenic acid, long-chain) and their blood pressure: INTERMAP study. Hypertension 50, 313-319.

20. Pietinen P, Paturi M, Reinivuo H, et al. (2010) FINDIET 2007 Survey: energy and nutrient intakes. Public Health Nutr 13, 920-924.

21. Pot GK, Prynne CJ, Roberts C, et al. (2012) National Diet and Nutrition Survey: fat and fatty acid intake from the first year of the rolling programme and comparison with previous surveys. Br J Nutr 107, 405-415.
22. Food Standards Agency (2009) Saturated fat campaign. http:// www.food.gov.uk/scotland/news-updates/news/pressreleases/ 2009/feb/launchsatfatcampaign (accessed June 2015).

23. Food Safety Authority of Ireland (2011) Scientific Recommendations for Healthy Eating Guidelines in Ireland. Dublin: FSAI. https://www.fsai.ie/recommendationsforhealthyeatingguide linesinireland.html (accessed June 2015).

24. Irish Universities Nutrition Alliance (2001) North/South Ireland Food Consumption Survey. http://www.iuna.net/ docs/NSIFCSummary.pdf (accessed June 2015).

25. Joyce T, Wallace AJ, McCarthy SN, et al. (2009) Intakes of total fat, saturated, monounsaturated and polyunsaturated fatty acids in Irish children, teenagers and adults. Public Health Nutr 12, 156-165.

26. Irish Universities Nutrition Alliance (2011) National Adult Nutrition Survey. Summary report. http://www.iuna.net/ wp-content/uploads/2010/12/National-Adult-Nutrition-SurveySummary-Report-March-2011.pdf (accessed June 2015).

27. Harrington KE, McGowan MJ, Kiely M, et al. (2001) Macronutrient intakes and food sources in Irish adults: findings of the North/South Ireland Food Consumption Survey. Public Health Nutr 4, 1051-1060.

28. Cashman KD, Muldowney S, McNulty B, et al. (2013) Vitamin D status of Irish adults: findings from the National Adult Nutrition Survey. Br J Nutr 109, 1248-1256.

29. Leite JC, Hearty AP, Nugent AP, et al. (2010) A method for assessing dietary intakes of $n-3$ long-chain polyunsaturated fatty acids and trans fatty acids in an Irish adult population. Int J Food Sci Nutr 61, 583-599.

30. Lennox A, Olson A \& Gay C (2010) Appendix K conversion of previous survey data to four-day estimates. https://www.gov. uk/government/uploads/system/uploads/attachment_data/ file/216496/dh_128552.pdf (accessed June 2015).

31. Food Standards Agency (2002) McCance and Widdowson's The Composition of Foods, 6th summary ed. Cambridge: Royal Society of Chemistry.

32. National Institute for Health and Welfare (2005) Fineli-Finnish Food Composition Database, release 5. http://www.fineli.fi/ (accessed June 2015).

33. Food Standards Australia New Zealand (2010) Nutrient tables for use in Australia. http://www.foodstandards.gov.au/ science/monitoringnutrients/nutrientables/Pages/default.aspx (accessed June 2015).

34. Black AE (2000) Critical evaluation of energy intake using the Goldberg cut-off for energy intake: basal metabolic. A practical guide to its calculation, use and limitations. Int J Obes Relat Metab Disord 24, 1119-1130.

35. Krebs-Smith SM, Kott PS \& Guenther PM (1989) Mean proportion and population proportion: two answers to the same question? J Am Diet Assoc 89, 671-676.

36. Scientific Advisory Committee on Nutrition (SACN) (2007) Update on Trans Fatty Acids and Health. Norwich: TSO.

37. Wearne SJ \& Day MJL (1999) Clues for the development of food-based dietary guidelines: how are dietary targets being achieved by UK consumers? Br J Nutr 81, S119-S126.

38. Central Statistics Office (2007) Census 2006: Principal Demographic Results. Dublin: The Stationery Office.

39. Kiely M, Flynn A, Harrington KE, et al. (2001) Sampling description and procedures used to conduct the North/South Ireland Food Consumption Survey. Public Health Nutr 4, 1029-1036.

40. Elmadfa I, Meyer A, Nowak V, et al. (2009) European nutrition and health report 2009. Forum Nutr 62, 1-405.

41. Micha R, Khatibzadeh S, Shi P, et al. (2014) Global, regional, and national consumption levels of dietary fats and oils in 
1990 and 2010: a systematic analysis including 266 country-specific nutrition surveys. BMJ 348, g2272.

42. Linseisen J, Welch AA, Ocke M, et al. (2009) Dietary fat intake in the European prospective investigation into cancer and nutrition: results from the 24-h dietary recalls. Eur J Clin Nutr 63, S61-S80.

43. The Department of Health and Children (2011) Your guide to healthy eating using the food pyramid. https://www.healthpromotion.ie/hp-files/docs/HPM00796.pdf (accessed June 2015).

44. Ericson U, Hellstrand S, Brunkwall L, et al. (2015) Food sources of fat may clarify the inconsistent role of dietary fat intake for incidence of type 2 diabetes. Am J Clin Nutr 101, $1065-1080$.

45. Sioen I, Devroe J, Inghelo P, et al. (2010) The influence of $n-3$ PUFA supplements and $n-3$ PUFA enriched foods on the $n-3$ LCPUFA intake of Flemish women. Lipids 45, 313-320.

46. Sioen I, Vyncke K, De Maeyer M, et al. (2013) Dietary intake and food sources of total and individual polyunsaturated fatty acids in the Belgian population over 15 years old. Lipids $\mathbf{4 8}$, 729-738.

47. Van Rossum CTM, Fransen HP, Verkaik-Kloosterman J, et al. (2011) Dutch National Food Consumption Survey 2007-2010. Diets of Children and Adults Aged 7-69 Years.
RIVM Report, no. 350050006/2011. Bilthoven: National Institute for Public Health and the Environment.

48. Harika RK, Eilander A, Alssema M, et al. (2013) Intake of fatty acids in general populations worldwide does not meet dietary recommendations to prevent coronary heart disease: a systematic review of data from 40 countries. Ann Nutr Metab 63, 229-238.

49. Mozaffarian D, Micha R \& Wallace S (2010) Effects on coronary heart disease of increasing polyunsaturated fat in place of saturated fat: a systematic review and meta-analysis of randomized controlled trials. PLOS Med 7, e1000252.

50. de Souza RJ, Mente A, Maroleanu A, et al. (2015) Intake of saturated and trans unsaturated fatty acids and risk of all-cause mortality, cardiovascular disease, and type 2 diabetes: systematic review and meta-analysis of observational studies. BMJ 351, h3978.

51. Heitmann BL, Lissner L \& Osler M. (2000) Do we eat less fat, or just report so? Int J Obes Relat Metab Disord 24, 435-442.

52. Sioen IA, Pynaert I, Matthys C, et al. (2006) Dietary intakes and food sources of fatty acids for Belgian women, focused on $n-6$ and $n-3$ polyunsaturated fatty acids. Lipids $\mathbf{4 1}, 415-422$.

53. Nelson M, Black AE, Morris JA, et al. (1989) Between- and within-subject variation in nutrient intake from infancy to old age: estimating the number of days required to rank dietary intakes with desired precision. Am J Clin Nutr 50, 155-167. 